\section{Valency and superconductors}

SIR-Tranquada et al. ${ }^{1}$ report X-ray absorption measurements on $\mathrm{Nd}_{2-x} \mathrm{Ce}_{x} \mathrm{CuO}_{4}$, on the basis of which they claim that some $\mathrm{Cu}^{2+}$ ions are converted to $\mathrm{Cu}^{+}$ions by cerium doping and also that cerium is mixed-valent. In my opinion, these claims, which are relevant to theories of high- $T_{\mathrm{c}}$ superconductivity ${ }^{2}$, should be reconsidered.

The technique used to determine valency involves the removal of a core electron. It is well known that, because of core-holeinduced changes in the final electronic state, the spectra may not reflect the true configuration of the initial state ${ }^{3}$. This issue has been discussed widely in the context of the valency of cerium in $\mathrm{CeO}_{2}$. We have shown ${ }^{4.5}$ that cerium is tetravalent in $\mathrm{CeO}_{2}$ and that the mixed valency proposed by others is the result of covalent mixing of $4 f$ levels in the valence band ${ }^{6}$. A similar situation might exist in $\mathrm{Nd}_{2-x} \mathrm{Ce}_{x}$ $\mathrm{CuO}_{4}$, in which case the $\mathrm{X}$-ray absorption data alone would not be sufficient to establish the mixed valency of cerium. We suggest that cerium is in fact tetravalent in this superconductor also; but one must then ask how the $\mathrm{Ce}^{4+}$ state is possible in a metallic environment, in view of established ideas to the contrary ${ }^{7.8}$ in terms of kondo interactions.

The valency of $\mathrm{Cu}$ is a more serious issue. There are no major differences between the X-ray-absorption spectral features of copper in $\mathrm{Nd}_{2} \mathrm{CuO}_{4}$ and those in cerium-doped specimens. Those small differences in lineshape and linewidth that might exist may be ascribed to greater $3 d$ hybridization in Ce-doped compounds and may be unrelated to $\mathrm{a} \mathrm{Cu}^{+}$component; it is commonly believed that hybridization in the ground state may cause such effects.

Thus, current understanding of the processes involved in the X-ray absorption method, particularly for copper $\mathrm{K}$-edge measurements, is not sufficient for any firm conclusions to be drawn regarding the valency of copper or cerium in these compounds on the basis of the data in ref. 1. Further experiments are required before the presence of $\mathrm{Cu}^{+}$or of mixedvalent cerium can be considered to be unambiguously established.

E.V. SAMPATHKUMARAN Tata Institute of Fundamental Research, Bombay 400005, India

TRANQUADA ET $A L$. REPLY-We agree with Sampathkumaran that further characterization of this material is required, but we stand by our previous analysis of the near-edge structures.

The changes observed at the copper $\mathbf{K}$ edge as a result of cerium doping are small on an absolute scale; they are quantitatively consistent, however, with the changes expected if one $\mathrm{Cu}^{2+}$ is converted to $\mathrm{Cu}^{+}$for each cerium atom that replaces a neodymium. They cannot be explained by a simple shift of the energy scale. It would be helpful to have good cluster calculations of the X-ray absorption spectra for different initial-state configurations, as there are no good model compounds for the cerium-doped material. Nevertheless, we believe that the changes cannot be explained by the creation of the $3 d^{9} 4 s$ configuration, as some have suggested, but instead result from a mixing of $3 d^{10}$ and $3 d^{9}$ states. Furthermore, our interpretation is consistent with the expectation that, as in $\mathrm{CuO}$, the first state to be filled on adding an electron should be the copper $3 d$ hole ${ }^{9}$. Sampathkumaran suggests that the changes are due to hybridization effects. If doping causes changes in the hybridization, then similar effects should be seen in the hole-doped compound $\mathrm{La}_{2-x} \mathrm{Sr}_{x} \mathrm{CuO}_{4}$, which we clearly do not see. The lattice parameters change by a very small amount with cerium doping $^{10}$, and it is therefore unclear why one should expect any change in hybridization.

Measurements have now also been performed at the oxygen $\mathrm{K}$ and copper $\mathrm{L}_{3}$ edges $^{11,12}$. The strong peak at the copper $\mathrm{L}_{3}$ edge is found to decrease with cerium doping, indicating a decrease in the density of copper $3 d$ holes. The sharp lowenergy peak at the oxygen $\mathbf{K}$ edge probably corresponds to a transition from the oxygen $1 s$ state to the copper $3 d$ state, which is allowed as a result of oxygen $2 p-$ copper $3 d$ hybridization. The situation appears very similar to that found in $\mathrm{NiO}$ (ref. 13). The alternative interpretation given by the authors of ref. 12 - that the oxygen $\mathrm{K}$-edge measurements indicate the presence of holes of a predominantly oxygen $2 p$ character - is difficult to reconcile with our copper $\mathrm{K}$-edge results.

We do not really differ with Sampathkumaran as far as the valency of cerium is concerned. We agree that the cerium added to $\mathrm{Nd}_{2} \mathrm{CuO}_{4}$ is very similar to that in $\mathrm{CeO}_{2}$, and do not object to covalentlybonded cerium being called tetravalent. The important point is that the cerium donates extra electronic charge to the

1. Tranquada, J.M., Heald, S.M., Moodenbaugh, A.R. Liang, G. \& Croft, M. Nature 337, 720-721 (1989).

Liang, G. \& Croft, M. Nature 337, 720-721
Rice, T.M. Nature 337, 686-687 (1989).

3. Sampathkumaran, E.V. et al. Phys. Rev. Lett. 54, 1067 1070 (1985).

4. Kaindl, G. et al. Phys. Rev. Lett. 58, 606-609 (1987)

5. Kaindl, G. et al. Phys. Rev. B38, 10174-10177 (1988)

6. Koelling, D.D. et al. Solid State Commun. 47, 227-230 (1983).

7. Kalkowski, G. et al. Phys. Rev. B32, 2717-2720 (1985)

8. Allen, J.W. \& Martin, R. Phys. Rev. Lett. 49, 1106-1110 (1982).

9. Ghijsen, J. et al. Phys. Rev. 838, 11322-11330 (1988)

10. Takagi, H., Uchida, S. \& Tokura, Y. Phys. Rev. Lett. 62 1197-1200 (1989)

11. Lin, C.L. et al. Phys. Rev. B (submitted).

12. Nücker, N. et al. Z. Phys. B (in the press)

13. Kuiper, P. et al. Phys. Rev. Lett, 62, 221-229 (1989) system, and that charge appears to go into copper $3 d$ holes.

J. M. TRANQUADA

S. M. HEALD

A. R. MOODENBAUGH

Brookhaven National Laboratory,

Upton, New York 11973, USA

G. LIANG

M. CROFT

Physics Department,

Rutgers University, Piscataway,

New Jersey 08855-0849, USA

\section{Not so fishy}

Sir_John Maddox's comment " Another red herring leads nowhere" on our recent paper $^{2}$ unfortunately contains some incorrect physics. Our work on anglecorrelated two-photon lines in 6-MeV-pernucleon collisions of $\mathrm{U}+\mathrm{Th}, \mathrm{U}+\mathrm{U}$ and $\mathrm{Th}+\mathrm{Th}$ provides evidence for approximately $180^{\circ}$-correlated two-photon lines up to about $1.1 \mathrm{MeV}$. Because the individual photons have approximately onehalf of this energy, they cannot give rise to electron-positron pairs, as is implied in Maddox's comment. In fact, it had been shown several years ago that if the electron-positron pair lines found at the Gesellschaft für Schwerionenforschung, Darmstadt (GSI) were due to external or internal pair conversion, the corresponding single $\gamma$-rays would have to stand out significantly in any single photon spectrum. Such protruding $\gamma$-rays have not been found ${ }^{3-5}$. Therefore, whatever the GSI electron-positron phenomenon may be, it is not simply "secondary products of single $\gamma$-rays released from nuclei put into high rotational states". At present, there does not seem to be any red herring, but simply unexplained scientific fact.

We wholeheartedly agree with Maddox that science sometimes needs to take risks and to use costly manpower and accelerators in order to progress. The results may not always be clear and their significance may take years of painstaking work to bear fruit. We are somewhat dismayed, though, to see the carefully done work on the GSI electron-positron phenomenon be put on a par with the initial studies on 'cold fusion'. In their style, the two studies appear not to belong in the same class.

W. E. MEYERHOF

K. DANZMANN

Department of Physics,

Stanford University

Stanford, California 94305-4060, USA

Nuclear Structure Research Laboratory,

University of Rochester,

Rochester, New York 14627, USA

1. Maddox, J. Nature 339, 253 (1989)

2. Danzmann, K. et al. Phys. Rev. Lett. 62, 2353-2356 (1989).

3. Clemente, M. et al. Phys. Lett. 137B, 41 (1984)

4. Bokemeyer, H. GSI preprint No. 84/43 (1984).

5. Cowan, T. thesis, Yale Univ. (1988) 